1Fundação Oswaldo Cruz (Fiocruz), Escola Nacional de Saúde Pública Sergio Arouca (Ensp) - Rio de Janeiro (RJ), Brasil. ligiagiovanella@gmail.com

2 Universidade Federal de Bahia (UFBA), Instituto de Saúde Coletiva (ISC) Salvador (BA), Brasil.

3 Universidade de São Paulo (USP), Faculdade de Saúde Pública (FSP) - São Paulo (SP), Brasil.

\section{A contribuição da Atenção Primária à Saúde na rede SUS de enfrentamento à Covid-19}

\author{
The contribution of Primary Health Care in the SUS network to face \\ Covid-19
}

Ligia Giovanella', Valentina Martufi², Diana Carolina Ruiz², Maria Helena Magalhães de Mendonça', Aylene Bousquat3, Rosana Aquino², Maria Guadalupe Medina²

DOI: $10.1590 / 0103-1104202113014$

RESUMO A centralidade no cuidado individual a casos graves descurou a abordagem populacional comunitária necessária ao enfrentamento da pandemia de Covid-19 no Sistema Único de Saúde (SUS). Neste ensaio, argumenta-se que a Estratégia Saúde da Família (ESF), com suas equipes multiprofissionais e enfoque comunitário e territorial, tem potencial para atuar na abordagem comunitária necessária ao enfrentamento da epidemia. A partir de experiências locais e internacionais, analisa quatro campos de atuação da Atenção Primária à Saúde (APS) no SUS no enfrentamento da Covid-19: vigilância nos territórios; cuidado individual dos casos confirmados e suspeitos de Covid-19; ação comunitária de apoio aos grupos vulneráveis; e continuidade dos cuidados rotineiros da APS. Reconhecem-se limites dessa atuação decorrentes de mudanças recentes na Política Nacional de Atenção Básica que afetam o modelo assistencial da vigilância em saúde. Conclui-se ser necessário ativar os atributos comunitários das equipes multiprofissionais da ESF e do Núcleo de Apoio à Saúde da Família; associar-se às iniciativas solidárias das organizações comunitárias e articular-se intersetorialmente; e garantir a continuidade das ações de promoção, prevenção e cuidado, criando novos processos de trabalho na vigilância em saúde, no apoio social e sanitário aos grupos vulneráveis e na continuidade da atenção rotineira para quem dela precisa.

PALAVRAS-CHAVE Atenção Primária à Saúde. Vigilância em saúde. Continuidade. Ação comunitária.

\begin{abstract}
The focus on individual care for severe cases neglected the community-centered approach required to cope with the Covid-19 pandemic in the Unified Health System (SUS) in Brazil. This essay argues that the Family Health Strategy (ESF), by means of its multi-professional teams and community and territorial orientation, is able to successfully develop the community approach required to deal with the pandemic. Inspired by local and international experiences, this essay analyzes four dimensions regarding SUS' Primary Health Care (PHC) work in the fighting against Covid-19: community-based health surveillance, individual care for confirmed and suspected cases of Covid-19, community mobilization to support vulnerable local groups, and continuity of APS care routine. Limitations are acknowledged due to recent changes in the National Policy of Primary Health Care impacting health surveillance care model. The conclusion iffor the need to: strengthen the community attributes of APS and Family Health Support multi-professional teams; collaborate with community organizations in initiatives of solidarity and articulate in an intersectoral way; guarantee the ongoing promotion, prevention and care actions by creating new working processes for health surveillance, social and health support for vulnerable groups, and for the continuity of the routine care for those in need.
\end{abstract}

KEYWORDS Primary Health Care. Health surveillance. Care continuity. Community participation. 


\section{Introdução}

A pandemia de Covid-19 alastra-se sem trégua pelo Brasil desde março de 2020, gerando uma crise sanitária e humanitária sem precedentes. A ausência de autoridade sanitária nacional e de coordenação e cooperação intergovernamental e a insuficiência e morosidade na alocação de recursos fazem com que as iniciativas de governos estaduais e municipais não sejam suficientes para enfrentar a pandemia. A centralidade das iniciativas de enfrentamento baseadas em cuidado individual de casos graves via criação de leitos de Unidade de Terapia Intensiva (UTI), hospitais de campanha e ações esparsas, ademais da negação da ciência e o descaso governamental federal, contribuíram para tal situação.

Enfrentar uma epidemia exige que se associe à atenção individual o cuidado centrado na comunidade, requerendo uma abordagem populacional. Essa junção é essencial para se combater a crise sanitária e humanitária atual' 1 . Sistemas de saúde fortemente baseados na Atenção Primária à Saúde (APS) podem ofertar esse cuidado integral e articulado, respondendo com mais efetividade às emergências ${ }^{2-4}$.

A APS tem papel crucial nessa necessária abordagem comunitária e de vigilância em saúde. A APS do Sistema Único de Saúde (SUS), em especial a Estratégia Saúde da Família (ESF), com suas equipes multiprofissionais e enfoque comunitário e territorial, que apresenta ao longo do tempo impactos positivos comprovados na saúde da população ${ }^{5}$, pode e deve atuar na abordagem comunitária necessária ao enfrentamento de qualquer epidemia e tem papel decisivo na rede assistencial de cuidados, no controle da epidemia e na continuidade do cuidado.

Nossas equipes de APS conhecem seus territórios, sua população, suas vulnerabilidades e, em geral, atuam na perspectiva da vigilância em saúde, o que é crucial para o controle do contágio. No entanto, é necessário reconhecer as fragilidades da APS no SUS. Desde o golpe parlamentar em 2016 e, especialmente, desde a Política Nacional de Atenção Básica (PNAB) de 2017, somaram-se novas dificuldades aos desafios sempre enfrentados ${ }^{6}$.

O modelo assistencial da APS brasileira, por meio de sua abordagem territorial e comunitária, vem sendo descaracterizado e se aproxima de um modelo de assistência individual, respondendo a problemas agudos, sem vínculo, continuidade, coordenação ou responsabilidade populacional 7 . Os efeitos do desfinanciamento agudo do SUS desde o golpe de 2016, aprofundado pelo governo Bolsonaro-Guedes, se revelavam já antes da pandemia por meio de desabastecimento de insumos e medicamentos essenciais, ausência ou precariedade na contratação de recursos humanos na APS e de imensa sobrecarga dos municípios, também em difícil situação financeira e responsáveis por mais de $30 \%$ do financiamento do SUS.

Além disso, a política econômica do Governo Bolsonaro-Guedes já afetara todos os determinantes sociais da saúde com piora de indicadores tais como aumento da mortalidade infantil e ressurgimento de outras doenças, como o sarampo, dengue e febre amarela ${ }^{8}$. Não obstante, iniciativas municipais já em curso mostram potencialidades da APS no enfrentamento da Covid-19 que podem e devem ser fortalecidas.

Assim, este ensaio tem como objetivo discutir a necessidade de fortalecimento da APS no SUS para o efetivo enfrentamento da pandemia de Covid-19 no Brasil. Apoia-se na ainda escassa literatura sobre esse recente evento, no aprendizado institucional e organizacional da APS brasileira e na experiência de pesquisa em APS das autoras. O artigo centra-se na organização da atenção primária, sem esquecer, contudo, que o cuidado individual e coletivo somente se realiza com serviços de APS bem integrados à rede assistencial, com canais de comunicação ágeis e abertos para a garantia do cuidado integral, conforme a necessidade de cada caso e população.

Inicia realçando a importância da APS no enfrentamento da pandemia, identifica eixos 
de atuação da APS a partir de experiências locais e internacionais, destaca a necessária articulação com os setores de vigilância em cada município e ilumina alguns dos desafios para o pleno desenvolvimento de ações de vigilância em saúde a partir das equipes da ESF em uma reflexão que objetiva articular os aspectos estruturais e conjunturais da crise anunciada.

\section{Por que a APS?}

Dois importantes aspectos devem ser considerados durante uma pandemia: o enfrentamento do agravo e a continuidade dos cuidados para o conjunto dos problemas de saúde que continuam acontecendo.

Por um lado, dada a capacidade exponencial de difusão da infecção, a identificação precoce e o isolamento dos casos pelos serviços de APS tornam-se fundamentais para reduzir a transmissibilidade. Cada caso identificado, com busca ativa de contatos próximos, isolamento em domicílio ou em locais apropriadamente organizados para isso, interrompe a transmissão, evitando inúmeros casos de Covid-19.

Por outro lado, analisar experiências anteriores de enfrentamento de epidemias nos auxilia a definir melhores estratégias de intervenção dos sistemas de saúde. Nas epidemias, se observa um excesso de mortes por outras causas que deixam de ser atendidas, que podem superar aquelas geradas pela própria doença.

No caso da epidemia de ebola, ficou demonstrado que a resposta sanitária global foi dependente do grau de competência da APS 9 . Essa mesma experiência de enfrentamento mostrou que o foco exclusivo na epidemia pode ter exercido efeitos desastrosos na morbidade e mortalidade, em curto e médio prazos, por outros problemas de saúde associados à redução do acesso e da continuidade do cuidado para os usuários com outras condições e agravos ${ }^{2}$.
Com relação à pandemia atual, além do impacto específico relacionado à Covid-19, já há estimativas de sobremortalidade por outras causas em distintos cenários de países de média e baixa renda em razão da redução de acesso e cobertura de ações usualmente ofertadas nos serviços de saúde. Estima-se que a redução do cuidado materno infantil durante a pandemia de Covid-19 em países de média e baixa rendas pode resultar em aumento de até $40 \%$ na mortalidade em menores de cinco anos e em até $30 \%$ na mortalidade materna ${ }^{10}$.

As diferentes formas de enfrentamento da pandemia da Covid-19 no âmbito da APS nos países do mundo foram condicionadas por modelos de organização desse nível de atenção e sua inserção nos sistemas nacionais de saúde, além dos contextos e de políticas locais. Com poucas exceções, pode-se dizer que, em razão da centralidade no cuidado hospitalar, perderam-se oportunidades de uma efetiva atuação da APS. Não obstante, diversas experiências e autores mostram a importância do fortalecimento da APS no enfrentamento da Covid-19.

Em Portugal, foram criadas unidades de APS comunitárias específicas - as 'Áreas Dedicadas Covid-19 Comunidade' - em cada Agrupamento de Centros de Saúde para atendimento de sintomáticos respiratórios do território"1. Na província de Wuhan, China, a maior parte dos 203 centros de APS realizaram ações para controle da Covid-19 envolvendo triagem e testes de casos suspeitos, monitoramento dos contatos e atenção clínica às pessoas em quarentena ${ }^{12}$. Em locais como Cuba e o estado de Kerala na Índia, o trabalho de agentes comunitários e equipes de APS nas comunidades contribuiu para a busca ativa de casos e contatos e para a identificação de pessoas em situação de vulnerabilidade ${ }^{4,13}$. Na Espanha, profissionais dos centros de APS foram deslocados para trabalho em hospitais, provocando desassistência na APS. A catástrofe da experiência italiana mostrou que a resposta à pandemia não pode ter foco apenas nos hospitais?. 


\section{Campos de atuação da APS no SUS no enfrentamento da Covid-19}

A APS tem, portanto, um papel essencial no enfrentamento da Covid-19, tanto agora, no momento da pandemia, como na continuidade da vigilância frente a outras ondas da doença e ao longo do tempo, pois existe risco de que se torne uma doença endêmica, pelo menos até que se tenha uma vacina efetiva.

No enfrentamento da pandemia, é necessário aliar cuidado individual e coletivo, i.e., cuidado oportuno de qualidade coordenado na rede e ativação dos atributos comunitários da APS. Neste momento, faz-se necessária a atuação integrada das unidades de saúde com os territórios, com a comunidade e com seus equipamentos sociais. É importante que a reorganização do processo de trabalho na APS no contexto da epidemia se faça de modo a preservar os seus atributos de acesso, longitudinalidade, coordenação do cuidado, abordagem familiar e abordagem comunitária ${ }^{\mathbf{1 4}}$.

Os modos de operação da APS devem ser adaptados a cada contexto e de maneira que não haja conflitos entre as ações. Isso vai requerer, por um lado, mudanças organizacionais em função de cada realidade local15 e, por outro, mobilização de recursos públicos, como dinheiro, pessoal e material. Demandará, também, recursos comunitários, via parceria com instituições atuantes no território e com a comunidade onde se instalam as equipes e unidades de saúde, assegurando o desenvolvimento das ações, potencializando habilidades e estimulando a solidariedade.
Urge:

- ativar os atributos comunitários das equipes multiprofissionais da ESF e do Núcleo de Apoio à Saúde da Família (Nasf).

- associar-se às iniciativas solidárias das organizações comunitárias e articular-se intersetorialmente para apoiar sua população em suas diversas vulnerabilidades.

- garantir a continuidade das ações de promoção, prevenção e cuidado, criando novos processos de trabalho na vigilância em saúde, no apoio social e sanitário aos grupos vulneráveis, na continuidade da atenção rotineira para quem dela precisa.

Defender a vida implica encontrar as melhores formas de enfrentar a epidemia no SUS a partir da APS.

Mesmo com todas as dificuldades, o SUS resiste. Iniciativas municipais e locais têm fortalecido a Atenção Primária à Saúde-Atenção Básica (APS-AB) tanto para tentar controlar o contágio nos territórios e prestar cuidado individual de casos suspeitos e confirmados de Covid-19 como para garantir a continuidade dos cuidados de rotina da $\mathrm{AB}$ com processos diversificados no território nacional.

As equipes de APS podem contribuir para a rede assistencial de cuidados e para a abordagem comunitária necessária ao enfrentamento da pandemia. Experiências locais de organização da APS para o enfrentamento da Covid-19 em diversos municípios sugerem que a atuação das equipes APS-AB na rede de enfrentamento à Covid-19 se organiza em quatro campos de ação integrados, com atuação das equipes da ESF, Saúde Bucal, Nasf, Agente Comunitário de Saúde (ACS) e agentes de endemias nos territórios, conforme sintetizado no quadro $1^{\mathbf{1 5}, 16}$. 
Quadro 1. Campos de atuação da Atenção Primária à Saúde no SUS na rede de enfrentamento da pandemia de Covid-19

\begin{tabular}{|c|c|}
\hline Eixo de atuação & Ações necessárias \\
\hline $\begin{array}{l}\text { Vigilância em saúde } \\
\text { nos territórios }\end{array}$ & $\begin{array}{l}\text { - Realizar ações de prevenção primária e secundária } \\
\text { - Identificação de casos e busca ativa de contatos } \\
\text { - Notificação de casos segundo definição atualizada } \\
\text { - Apoio ao isolamento domiciliar de casos e contatos } \\
\text { - Acompanhamento cotidiano à distância dos casos em cuidado domiciliar } \\
\text { - Apoio na vigilância em lares para idosos e outras instituições de longa permanência em seu } \\
\text { território } \\
\text { - Prover informação e educação em saúde na comunidade e nos serviços essenciais } \\
\text { - Potencializar recursos de comunicação coletiva existentes na comunidade tais como rádios } \\
\text { comunitárias, grupos de mensagens, carro de som etc. } \\
\text { - Informar sobre situação epidemiológica do território e medidas de proteção, como distancia- } \\
\text { mento social, uso de máscaras, lavagem mãos etc. } \\
\text { - Testar casos, contatos e profissionais de saúde }\end{array}$ \\
\hline $\begin{array}{l}\text { Cuidado individual } \\
\text { dos casos confir- } \\
\text { mados e suspeitos } \\
\text { Covid-19 }\end{array}$ & $\begin{array}{l}\text { - Organizar os fluxos de atenção } \\
\text { - Separar os fluxos de atenção dos sintomáticos respiratórios/casos suspeitos, dos pacientes } \\
\text { com outros problemas/necessidades } \\
\text { - Reduzir ao mínimo o número de profissionais que entram em contato com cada caso suspei- } \\
\text { to/sintomáticos respiratórios } \\
\text { - Adequar a infraestrutura } \\
\text { - Cuidar dos pacientes com quadros leves } \\
\text { - A avaliação da história e da clínica deve ser oportuna e rápida } \\
\text { - Classificar a gravidade segundo protocolo clínico } \\
\text { - Disponibilizar equipamentos tais como oxímetro, termômetro infravermelho, oxigênio na } \\
\text { UBS } \\
\text { - Cuidar conforme os protocolos mais atualizados e em base ao melhor conhecimento cientí- } \\
\text { fico } \\
\text { - Telemonitoramento pela equipe de casos e contatos: recomenda-se contato diário, de prefe- } \\
\text { rência de } 12 / 12 \text { hs } \\
\text { - Prover EPIs apropriados para todos os profissionais conforme atividade realizada e capacita- } \\
\text { ção sobre utilização adequada } \\
\text { - Garantir o encaminhamento oportuno daqueles que necessitem de cuidados de outros níveis } \\
\text { de atenção } \\
\text { - Garantir transporte oportuno e específico para Covid-19 } \\
\text { - Prover estabilização clínica até o transporte chegar } \\
\text { - Teleatendimento disponibilizando telefone de contato para os usuários } \\
\text { - Linha de cuidado começa por telefone específico para atenção aos sintomáticos respiratórios } \\
\text { com comunicação às equipes de casos de sua área para acompanhamento diário por telefone } \\
\text { - Todas as equipes e UBS precisam ser providas com telefones e acesso à internet para facili- } \\
\text { tar o atendimento não presencial aos usuários } \\
\text { - Integração da APS com Centrais de Atendimento à população, como SESRJ: } 160 \text { e Centrais } \\
\text { municipais, para acompanhamento dos casos suspeitos pelas equipes APS } \\
\text { - Capacitar profissionais para o cuidado }\end{array}$ \\
\hline $\begin{array}{l}\text { Apoio aos grupos vul- } \\
\text { neráveis no território } \\
\text { por sua situação de } \\
\text { saúde ou social }\end{array}$ & $\begin{array}{l}\text { - Articular a iniciativas comunitárias e promover a atuação intersetorial } \\
\text { - Mobilizar lideranças e organizações sociais } \\
\text { - Divulgar informações e esclarecer dúvidas sobre medidas de prevenção } \\
\text { - Apoiar a distribuição de recursos doados, materiais de higiene, cestas básicas etc. } \\
\text { - Mapear usuários de maior risco para a Covid-19 tais como idosos, crônicos, pessoas em } \\
\text { extrema pobreza ou com insegurança alimentar } \\
\text { - Reforçar medidas de prevenção e garantir recursos para que se estabeleçam as condições de } \\
\text { ficar em casa } \\
\text { - Acionar as redes de apoio social } \\
\text { - Articular se com profissionais do Cras para cadastro programas sociais } \\
\text { - Monitorar problemas de violência doméstica } \\
\text { Qualquer profissional da equipe pode se encarregar destas ações, incluindo ACS, agentes de ende- } \\
\text { mias, equipes de saúde bucal, profissionais dos Nasf. }\end{array}$ \\
\hline
\end{tabular}


Quadro 1. (cont.)

\begin{tabular}{ll}
\hline Eixo de atuação & Ações necessárias \\
\hline Continuidade dos & - Dar continuidade aos cuidados rotineiros \\
cuidados rotineiros & - Pré-natal, atenção aos hipertensos, diabéticos, vacinação etc. \\
da APS & - Elaborar listas de pacientes crônicos que necessitam de cuidado contínuo \\
& - Desenvolver novas formas de cuidado cotidiano à distância: disponibilidade de acesso à \\
& internet, WhatsApp individual e grupos de usuários, telefone, teleconsulta por vídeo, telemo- \\
& nitoramento \\
& - Visitas peridomiciliares dos ACS para acompanhamento e informação \\
& - Permanecer de porta aberta, mas com restrições \\
& - Garantir a continuidade da assistência farmacêutica \\
& - Organizar a distribuição domiciliar dos medicamentos pelo ACS \\
& - Desenvolver formas de receitas eletrônicas com certificação digital
\end{tabular}

Para ação efetiva, disponibilizar INTERNET PARA TODOS: profissionais de saúde e população. O poder público deve instalar Wi-Fi em cada bairro e comunidade, articular com operadoras para ampliar a internet de todos os cidadãos que têm contas telefônicas pré ou pós pagas; no mínimo articular para disponibilizar maior acesso, em minutos, mensagens e internet, para todos os profissionais, incluindo todos os ACS.

Fonte: Elaboração própria com base em Medina ${ }^{\mathbf{1 6}}$, Engstrom ${ }^{\mathbf{1 5}}$

\section{Vigilância em saúde nos territórios}

O modelo assistencial de vigilância da saúde que fundamenta a ESF deve orientar o enfrentamento da pandemia. A vigilância da saúde contempla o uso articulado da epidemiologia e das ciências sociais para a análise da situação da saúde, planejamento e organização das práticas num território específico. Integra atividades individuais e coletivas, ações setoriais, intersetoriais, de promoção da saúde, prevenção de riscos e doenças, e de cuidado ${ }^{17,18}$. Diversos municípios vêm fortalecendo a vigilância em saúde nos territórios com a participação da APS no enfrentamento da Covid-19, ainda que pesquisas indiquem que a vigilância da saúde ainda não é o modelo dominante na $\mathrm{ESF}^{\mathbf{1 9}, 20}$ e que existam fragilidades importantes na integração entre os setores de vigilância epidemiológica e as equipes de APS associadas à falta de comunicação e planejamento conjunto entre os dois setores, além de insuficiente capacitação em vigilância epidemiológica, dentre outros fatores ${ }^{21,22}$.

Com a finalidade de bloquear e reduzir o risco de expansão da epidemia, a vigilância em saúde implica coordenar, no território, ações de prevenção primária e secundária à Covid-19, como identificação de casos, testagem e busca ativa de contatos, apoio ao isolamento domiciliar de casos e contatos; notificação de casos; e ações de educação em saúde que potencializam recursos de comunicação coletiva existentes na comunidade tais como rádios comunitárias, grupos de mensagens, carro de som.

Diversos municípios fortaleceram a vigilância em saúde nos territórios criando Grupos de Trabalho (GTs) com a participação da APS. A Secretaria Municipal de Saúde de Belo Horizonte (MG) criou um GT com o propósito de discutir e definir ações conjuntas entre a áreas de vigilância e de assistência à saúde e de produzir notas técnicas, fluxos e protoco$\operatorname{los}^{23}$. Similarmente, a Clínica de Família Zilda Arns, situada no Complexo do Alemão (RJ), criou quatro GTs para enfrentar a pandemia, entre os quais um GT de Telemonitoramento Covid-19. Os profissionais integrantes desse GT desenvolveram, por iniciativa própria, um painel para apoiar a vigilância, atualizado diariamente pela equipe e diretamente conectado ao e-SUS de vigilância epidemiológica, permitindo a notificação ${ }^{24}$.

Para vigilância e controle efetivos do contágio, a experiência internacional tem evidenciado que uma ação fundamental é a testagem 
molecular oportuna por RT-PCR para a identificação de casos e busca ativa de contatos. $\mathrm{O}$ rastreamento de contatos e a respectiva quarentena foram um componente central da estratégia de resposta do Vietnã, que, até julho, apresentou poucos casos e nenhum óbito por Covid-19. O rastreamento de contatos é uma iniciativa abrangente apoiada por ampla rede de centros provinciais e distritais de controle de doenças que conta com 11.000 centros comunitários de saúde. Contatos de casos confirmados foram rastreados e testados, incluindo os contatos dos contatos, com isolamento de todos os positivos ${ }^{25}$.

$\mathrm{O}$ apoio ao isolamento domiciliar de casos, o rastreamento de contatos, o incentivo à quarentena dos contatos e o acompanhamento cotidiano à distância dos casos em cuidado domiciliar são ações que podem ser realizadas eficazmente pela APS ${ }^{26}$. Para o efetivo apoio ao isolamento e à quarentena de contatos, é necessário que a gestão pública disponibilize espaços comunitários específicos quando as condições do domicílio não permitirem.

Entre as atividades de educação em saúde, a conscientização sobre a necessidade de uso de máscaras por todas as pessoas é uma tarefa urgente. Há consenso que o uso de máscaras por todos na comunidade contribui para a efetiva redução do contágio, se as máscaras forem usadas pela grande maioria das pessoas, pois o contágio ocorre também a partir de pessoas assintomáticas ${ }^{27}$.

Em Florianópolis (SC), protocolos estabelecidos determinam que, para as pessoas identificadas por telemonitoramento com sintomas há menos de sete dias, deve ser realizado teste molecular RT-PCR no domicílio pelas equipes APS treinadas ${ }^{28}$. Igualmente crítica é a testagem de profissionais de saúde devido ao alto potencial de contágio, grupo priorizado em municípios como Belo Horizonte ${ }^{23}$ e Canaã dos Carajás (PA), onde foi montado monitoramento clínico exclusivo para profissionais de saúde e usuários com comorbidades ${ }^{29}$.

A testagem somente será efetiva se acompanhada por busca ativa de casos suspeitos e contatos com o objetivo de identificar e isolar precocemente, como tem feito a APS em Nova Lima (MG) e em Sobral (CE). Os ACS indagam quanto à presença de sinais e sintomas de gripe entre as famílias de sua microárea por meio de contatos telefônicos ou por aplicativo de mensagens em Nova Lima ${ }^{30}$. Em Sobral, os ACS planejam e realizam visitas peridomiciliares para identificar precocemente sinais de gravidade e prover orientações para isolamento domiciliar e adotar medidas preventivas para evitar a disseminação ${ }^{31}$. Em Nova Lima, os ACS contribuem, também, para o monitoramento de estabelecimentos comerciais que não estejam seguindo as determinações municipais, acionando a vigilância sanitária municipal quando necessário ${ }^{30}$.

As equipes de APS têm potencial para prover informação e educação em saúde à comunidade e aos serviços essenciais, como farmácias, mercados etc., e combater fake news, aproveitando recursos de comunicação coletivas existentes - rádios comunitárias, grupos de mensagens, carro de som etc. - com o objetivo de abordar a situação epidemiológica do território e adotar medidas de proteção - distanciamento social, uso de máscaras, lavagem de mãos. Foi assim que uma Unidade Básica de Saúde (UBS) rural do sertão pernambucano estabeleceu comunicação contínua com a comunidade: por meio de um programa radiofônico criado pelos profissionais da UBS e semanalmente divulgado pelo WhatsApp para os usuários e de um canal de comunicação aberto para esclarecer dúvidas relacionadas à pandemia e ao funcionamento da $\mathrm{UBS}^{32}$.

Em Londrina (PR), profissionais das equipes da ESF e do Nasf, afastados do trabalho rotineiro por pertencerem a grupos de risco, colaboraram remotamente com professores e estudantes da Universidade Estadual de Londrina em projeto para prover orientações para trabalhadores dos municípios da região sobre medidas de proteção e prevenção à Covid-19 no trabalho. A iniciativa alcançou condomínios comerciais e residenciais, salões de beleza, escolas, construção civil e indústrias, 
quando 109 multiplicadores repassaram treinamentos para 2.500 trabalhadores ${ }^{33}$. Em Recife, esforços direcionados à educação em saúde da população foram liderados por profissionais do Nasf em salas de espera e triagem, salas de vacina e filas no entorno das $\mathrm{UBS}^{34}$.

Além disso, as equipes de APS podem apoiar a vigilância em instituições de longa permanência, como os lares de idosos e casas de acolhimento institucional em seus territórios. Esses estabelecimentos precisam implantar cuidados especiais e uma vigilância intensificada, dado que aglomeram pessoas vulneráveis e são focos de contágio frequente - na Espanha, metade das mortes ocorreu em lares de idosos -, o que tem sido feito pelas equipes de APS nos municípios de Nova Lima ${ }^{30}$ e Belo Horizonte ${ }^{23}$, por exemplo.

\section{Cuidado individual dos casos confirmados e suspeitos de Covid-19}

Outra responsabilidade das equipes de APS é o cuidado individual dos casos confirmados e suspeitos de Covid-19, organizando fluxos separados de atenção para sintomáticos respiratórios ou casos suspeitos, cuidando dos pacientes com quadros leves e garantindo o encaminhamento oportuno daqueles que necessitem de cuidados de outros níveis de atenção. Isso, com o emprego de telemonitoramento pela equipe de casos e contatos e de teleatendimento via disponibilização de telefone de contato para os usuários.

A separação entre os fluxos de atenção dos sintomáticos respiratórios e dos pacientes com outros problemas ou necessidades tem sido realizada de diversas maneiras. Em UBS maiores, foram separados fluxos no interior das UBS, com primeiro atendimento fora da UBS e corredores e salas de atendimento e equipes de profissionais separadas para casos suspeitos. Em outros municípios, foram instaladas tendas no espaço exterior às UBS ou definidos estabelecimentos específicos para $o$ atendimento aos sintomáticos respiratórios que necessitam de cuidado presencial.
Por exemplo, o município de Teresina (PI) separou 25 de suas 90 UBS para atendimento ao Covid-19, e o de Canaã dos Carajás definiu unidade de referência com ambulância disponível para realizar a transferência dos casos graves para o hospital de campanha ${ }^{29}$. Nos lugares onde a infraestrutura das UBS permitiu divisão de fluxos internos, foram montadas tendas de triagem fora das unidades para determinar qual porta de entrada - Covid ou Não-Covid - cada usuário deveria utilizar, como em Florianópolis ${ }^{28}$, Sobral ${ }^{\mathbf{3 1}}$ e Belo Horizonte ${ }^{23}$.

A opção pela criação de polos de atenção à Covid-19 especificamente para atender aos casos suspeitos, realizar manejo inicial e facilitar o acesso à internação quando necessário deve ser acompanhada pela comunicação efetiva com as UBS para que possam acompanhar e fazer a vigilância em seus territórios ${ }^{35}$.

O cuidado individual de qualidade pelas equipes é condicionado à sua integração à rede e à garantia de transporte oportuno e específico para Covid-19, como foi feito em Belo Horizonte ${ }^{23}$, onde o transporte foi integrado à regulação de leitos, garantindo o acesso rápido ao cuidado hospitalar, com fluxos de referência e contrarreferência bem estabelecidos.

Outra estratégia para reduzir o contágio nas UBS é o teleatendimento via disponibilização de telefone de contato específico para atenção aos sintomáticos respiratórios, como foi feito em Florianópolis ${ }^{28}$. Isso pode ser realizado pela integração da APS com Centrais de Atendimento telefônico à população estaduais ou municipais para identificar os casos suspeitos em seus domicílios e comunicar às equipes casos de sua área para acompanhamento diário por telefone, tentando evitar viagens desnecessárias para as UBS. Além dos profissionais da APS, especialistas da rede secundária do SUS podem contribuir para o teleatendimento, como ocorreu em Belo Horizonte e Florianópolis, pois seus atendimentos foram reduzidos em decorrência das medidas de distanciamento social23,28. Em Florianópolis, foram também recrutados para essa função os profissionais das ESB e do Nasf ${ }^{\mathbf{2 8}}$. 
Para que o telemonitoramento e o teleatendimento possam ser viabilizados, é necessário prover telefones e acesso à internet para as equipes, facilitando o atendimento não presencial aos usuários. Em Florianópolis, já antes da pandemia, o município investira em um serviço de atendimento pré-clínico denominado 'Alô saúde Floripa' para prover as equipes da ESF de smartphone, chip pós-pago e acesso à plataforma WhatsApp Business. A chegada da pandemia demandou o fortalecimento dessa iniciativa, que incluiu ampliação da divulgação dos números de atendimento à população ${ }^{28}$.

A separação de fluxos não se refere somente aos espaços físicos, mas também aos recursos humanos envolvidos nos atendimentos. Recomenda-se, portanto, reduzir ao mínimo o número de profissionais que entram em contato com cada caso sintomático respiratório ou suspeito de Covid-19. Além disso, é recomendável que os profissionais dedicados ao atendimento de pacientes Covid-19 sejam oportunamente capacitados e que se invista na contratação de profissionais adicionais para atender aos fluxos separadamente, como em Canaã dos Carajás ${ }^{29}$. Dadas as restrições do distanciamento social, a SMS de Belo Horizonte organizou capacitações online por meio de vídeos e webconferências para gerentes e profissionais de saúde ${ }^{23}$.

A APS pode cuidar dos pacientes com quadros leves realizando uma avaliação da história e da clínica, oportuna e rápida, classificando a gravidade segundo protocolos clínicos e cuidando conforme protocolos atualizados com base no melhor conhecimento científico. Para essa atuação, é preciso disponibilizar nas UBS os equipamentos e os insumos necessários para a atenção aos casos suspeitos, como oxímetro, termômetro infravermelho, oxigênio, além de garantir Equipamentos de Proteção Individual (EPI) apropriados para todos os profissionais, acompanhados de capacitação sobre utilização adequada. Prover EPI para os profissionais de saúde foi um desafio para todos os serviços de saúde no início da pandemia devido à explosão do consumo e à dificuldade em encontrar fornecedores. Em Belo Horizonte, as áreas técnicas e de compras juntaram esforços para facilitar a aquisição dos insumos com maior agilidade e transparência ${ }^{23}$. Em Sobral, indústrias locais redirecionaram processos para a produção de EPIs ${ }^{31}$.

O cuidado individual inclui acompanhamento sistemático dos casos com boa comunicação e informações sobre a doença e sinais de agravamento, permitindo a procura oportuna dos serviços de saúde e oxigenioterapia precoce. Os casos leves e os contatos devem ser seguidos pelas equipes de APS por meio de telemonitoramento. Recomenda-se contato, preferencialmente diário, com telemonitoramento a cada $24 \mathrm{hs}$ ou $48 \mathrm{hs}$, dependendo da gravidade do caso $^{29,30}$. Vários profissionais envolvidos na APS podem contribuir para a realização de telemonitoramento, inclusive as equipes do Nasf ${ }^{34}$, Saúde Bucal e ACS ${ }^{30}$.

Os casos moderados e graves devem ser encaminhados oportunamente para os serviços de referência por transporte oportuno e específico para Covid-19; devendo ser proporcionadas condições para que aos profissionais de APS possam prover a estabilização clínica até o transporte chegar.

\section{Ação comunitária de apoio aos grupos vulneráveis no território por sua situação de saúde ou social}

Por sua estreita relação com o território, a APS pode articular iniciativas comunitárias e promover a atuação intersetorial para enfrentar os aspectos sociais da pandemia. Por meio da mobilização de lideranças e organizações comunitárias, pode-se conseguir apoio e colaboração da comunidade nas ações de enfrentamento, divulgar informações e esclarecer dúvidas sobre medidas de prevenção, identificar lares com possíveis problemas, como violência doméstica, e apoiar a distribuição de recursos doados.

A interação dos movimentos sociais com os serviços de saúde, especialmente mediados pelos ACS, facilita o mapeamento e apoio aos 
usuários de maior risco para a Covid-19, i.e., idosos, crônicos, pessoas em extrema pobreza ou com insegurança alimentar, para reforçar as medidas de prevenção e garantir recursos para que se estabeleçam as condições de ficar em casa, acionando as redes de apoio social, o cadastro em programas e o direcionamento de cestas básicas.

Nesse sentido, o GT Comunitário estabelecido pela Clínica de Família Zilda Arns (RJ) juntou esforços ao Gabinete de Crise do Complexo do Alemão, integrado por líderes comunitários dos três principais dispositivos sociais da área - o Coletivo Papo Reto, o jornal 'Voz da Comunidade' e o Coletivo Mulheres em Ação pelo Alemão. A parceria desenvolveu ações de comunicação e educação em saúde, organizou a distribuição de doações, cestas básicas e kits de higiene, além de ter articulado com outras organizações comunitárias e outras unidades de APS do Complexo do Alemão ${ }^{36}$.

Em Niterói, a APS colaborou com os Centros de Referência de Assistência Social (Cras) e com as escolas públicas para o mapeamento de população vulnerável para medidas de proteção social e para a disseminação de insumos e informações de prevenção do contágio ${ }^{37}$. Em Florianópolis, a articulação intersetorial e com a sociedade civil foi realizada juntamente com a Secretaria de Assistência Social e com o projeto 'Somar Floripa', constituído por uma rede de organizações voluntárias ${ }^{28}$. A Secretaria Municipal de Saúde de Belo Horizonte se mobilizou para cuidar da população em situação de rua, criando um serviço provisório e emergencial em parceria com a política pública de assistência social e com o Serviço Social do Comércio, disponibilizando 260 acomodações individuais com banheiro privativo, entre outras iniciativas ${ }^{23}$. Em Nova Lima, as equipes de APS foram orientadas a identificar os usuários de maior vulnerabilidade em seu território e desenvolver planos de suporte em colaboração com outros atores sociais ${ }^{\mathbf{3 0}}$.

Qualquer profissional das equipes de APS, com destaque para os ACS, pode liderar tais iniciativas e aproveitar a disponibilidade de profissionais cuja carga laboral foi diminuída, como foi a das equipes de saúde bucal e de profissionais do Nasf.

\section{Continuidade dos cuidados rotineiros da APS}

Ao contrário do que se praticou em várias experiências internacionais e nacionais, onde os serviços de APS foram suspensos quando chegou a pandemia ${ }^{9,23}$, é fundamental dar continuidade aos cuidados rotineiros da APS, pois as necessidades permanecem e corre-se o risco de os quadros se agravarem e de o sofrimento e a mortalidade aumentarem por outras causas.

Após a chegada da pandemia ao Brasil, dadas as muitas incertezas e o medo real do contágio, observou-se a suspensão de atividades em UBS e uma importante queda no número de atendimentos na APS, cuja continuidade poderia levar a complicações e problemas por falta de cuidado aos portadores de doenças crônicas ${ }^{23}$. Grávidas, crianças menores de cinco anos, hipertensos e diabéticos continuam precisando de atenção e seguimento, ao mesmo tempo que, em parte, integram os grupos de maior risco para Covid-19.

Para a continuidade da atenção, torna-se importante estabelecer novas formas de cuidado cotidiano à distância por meio de telefones e aplicativos para seguimento individual e de grupos de usuários pelo teleatendimento. A chave é a UBS permanecer de portas abertas, atendendo aos casos necessários, mas com restrições, resolvendo o que for possível à distância e reduzindo os atendimentos presenciais. Os ACS podem apoiar famílias e acompanhar grupos por meio de visitas peridomiciliares. A possibilidade de realização de visitas domiciliares por enfermeiros e médicos é uma opção que deve ser considerada para pacientes de maior risco.

Reforça-se aqui a necessidade de garantir vacinação de rotina de crianças e gestantes sem expor a população à Covid-19. Pode-se analisar a viabilidade de realizar vacinação em local fora da UBS, quando a vacinação na UBS possa comprometer a segurança dos grupos a vacinar. 
Em Salvador (BA), em unidade docente-assistencial da Escola Baiana de Medicina e Saúde Pública (EBMSP), a lista de pacientes acompanhados pela unidade foi revisada para determinar critérios de prioridade para teleconsultas, com realização do teleatendimento por meio de aplicativo e eventual agendamento de pacientes na UBS, conforme a necessida$\mathrm{de}^{38}$. Em Nova Lima, para dar continuidade ao cuidado dos crônicos, priorizaram-se as visitas domiciliares da equipe após o teleatendimento, deixando as idas às UBS para casos de maior necessidade ${ }^{30}$. Em Vitória (ES), uma iniciativa análoga foi liderada remotamente pelos ACS - que tinham sido proibidos de realizar visitas domiciliares por decreto municipal -, apoiados pelas equipes do Nasf $\mathbf{3 9}$.

Em Recife (PE), os profissionais do Nasf foram ativos no apoio às ações rotineiras da APS, incluindo a campanha de vacinação para gripe e atendimento dos casos de Covid-1934. Em Sobral, optou-se por realocar os profissionais de centros de especialidades médicas e de reabilitação que ficaram fechados por causa da pandemia para fortalecer a provisão de serviços rotineiros de APS ${ }^{31}$.

Cuidados em saúde mental na situação de pandemia necessitam especial atenção. Devem ser continuados e ampliados, uma vez que as medidas de distanciamento social podem produzir sofrimento mental e que os profissionais de saúde sobrecarregados necessitam de apoio. Profissionais do Nasf podem apoiar com consultas à distância, e serviços de saúde mental podem ser apoiados pela APS.

Em Itabuna (BA), uma integração entre as UBS e os serviços de saúde mental permitiu que os usuários de saúde mental pudessem continuar renovando suas prescrições nas UBS, o que diminuiu a necessidade de deslocamento até os centros especializados. Nesse cenário, as equipes ESF ficaram encarregadas de manejar atendimentos eletivos de saúde mental procurados por ansiedade leve ou distúrbios de relacionamento interpessoal, encaminhando para os serviços especializados somente os casos mais graves. Essa integração permitiu importante aproximação de usuários dos serviços de saúde mental aos serviços de APS, os quais raramente utilizavam antes da pandemia ${ }^{40}$.

A continuidade da assistência farmacêutica pode ser garantida com listas de pacientes crônicos que necessitam de tratamento contínuo para organizar a distribuição domiciliar dos medicamentos pelo ACS, como é feito em Canaã dos Carajás ${ }^{29}$. Pode-se, também, adotar formas de receitas eletrônicas com certificação digital para evitar visitas desnecessárias às UBS, como na experiência de Florianópolis, onde se estabeleceram normativas para que a rede de farmácias aceitasse as receitas de medicamentos comuns e antibióticos feitas por vídeo chamada, além de acordos com laboratórios para que aceitassem pedidos de exames em formato eletrônico ${ }^{\mathbf{2 8}}$.

A continuidade do trabalho do ACS é imprescindível para a abordagem comunitária da pandemia nos quatro eixos: vigilância, apoio a grupos vulneráveis, continuidade do acompanhamento das famílias por meio de visitas peridomiciliares e comunicação à distância. Para isso, é necessário promover atividades de educação continuada do ACS e garantir EPI adequado para a sua segurança, conforme $o$ tipo de atividade desenvolvida.

Para o desenvolvimento desse conjunto de ações de forma efetiva e eficiente, é necessário prover capacitação de todos os profissionais das equipes sobre enfrentamento da Covid-19 e sobre o uso de equipamentos de proteção individual adequado a cada tipo de atividade realizada.

\section{Considerações finais}

A atuação das equipes de APS é crucial em todos os estágios da pandemia. Se, em um primeiro momento, oportunidades de atuação efetiva da APS foram perdidas por ausência de autoridade sanitária nacional e de diretrizes nacionais claras, gerando suspensão de atividades e centralidade no cuidado hospitalar 
intensivo, no decurso da pandemia, em muitas localidades, as ações vêm sendo retomadas, como mostram as experiências aqui relatadas.

A pandemia prossegue com diferentes ritmos e estágios no vasto e desigual território nacional. Para continuação da desaceleração dos casos e retomada de atividades, é fundamental a intensificação da vigilância em saúde com a participação das equipes de APS, de forma a prevenir novas ondas da infecção. Mas há desafios a superar.

A atuação articulada da ESF e a vigilância em saúde no controle da Covid-19 requerem ações de capacitação dos profissionais das equipes da ESF que superem o simples repasse de informações técnicas clínicas e contribuam para a reflexão sobre o modelo de atenção, a participação social e a ação comunitária com uso dos dados epidemiológicos e sociais produzidos no nível local. É relevante que os diversos agentes envolvidos consigam operar conceitualmente o modelo de vigilância em saúde em suas diversas dimensões.

É imprescindível, portanto, ativar ainda mais os atributos comunitários das equipes multiprofissionais da ESF e do Nasf; associar-se às iniciativas solidárias das organizações comunitárias e articular-se intersetorialmente para apoiar a população em suas diversas vulnerabilidades; e garantir a continuidade das ações de promoção, prevenção e cuidado, criando novos processos de trabalho na vigilância em saúde e no apoio social e sanitário voltados aos grupos vulneráveis, para a continuidade da atenção rotineira para quem dela precisa.
Sem dúvida, a atuação da APS somente se efetiva plenamente em uma rede integrada ${ }^{41}$. Sem acesso aos serviços hospitalares para casos intermediários e graves, o diagnóstico precoce proporcionado pela APS não se concretiza em cuidado oportuno.

Mais que nunca, é importante continuar atendendo às pessoas, trabalhar em equipe, ainda que à distância, para garantir a continuidade da atenção com forte vínculo, pois, frente às incertezas e distanciamento social, estamos todos mais fragilizados.

O enfrentamento da pandemia tem revelado a distribuição desigual de serviços de saúde, exigindo a construção de redes assistenciais regionalizadas e o fortalecimento da autoridade sanitária estadual, o que pode ser um legado positivo para o SUS. Esses são tempos incertos que exigem reinventar processos de trabalho orientados a cada contexto, estabelecer novos fluxos, fortalecer redes, exercitar a solidariedade.

\section{Colaboradoras}

Giovanella L (0000-0002-6522-545X)*, Martufi V (0000-0001-6120-0629)*, Ruiz DC (0000-0002-0939-4881)*, Mendonça MHM (0000-0002-3917-9103)*, Bousquat A (0000-0003-2701-1570)*, Aquino R (00000003-3906-5170)* e Medina GM (00000001-7283-2947)* contribuíram igualmente para a elaboração do manuscrito. 


\section{Referências}

1. Nacoti M, Ciocca A, Giupponi A, et al. At the Epicenter of the Covid-19 Pandemic and Humanitarian Crises in Italy: Changing Perspectives on Preparation and Mitigation. NEJM Catal. 2020 [acesso em 2020 abr 22]; 1(2):1-5. Disponível em: https://catalyst.nejm.org/doi/ full/10.1056/CAT.20.0080\%0Ahttps://catalyst.nejm. org/doi/abs/10.1056/CAT.20.0080.

2. Dunlop C, Howe A, Li D, et al. The coronavirus outbreak: The central role of primary care in emergency preparedness and response. BJGP Open. 2020 [acesso em 2020 maio 28]; 4(1). Disponível em: https://bjgpopen. org/content/4/1/bjgpopen20X101041.

3. Redwood-Campbell L, Abrahams J. Primary health care and disasters - The current state of the literature: What we know, gaps and next steps. Prehosp Disaster Med. 2011; 26(3):184-91.

4. Sundararaman T. Health systems preparedness for COVID-19 pandemic. Indian J Public Health. 2020 [acesso em 2020 jul 10]; 64(6):91. Disponível em: http://www. ijph.in/text.asp?2020/64/6/91/285624.

5. Aquino R, Oliveira NF, Barreto ML. Impact of the Family Health Program on Infant Mortality in Brazilian Municipalities. Am J Public Health. 2009; 99(1):87-93.

6. Rede de Pesquisa em Atenção Primária à Saúde da Abrasco. Contribuição dos pesquisadores da Rede APS ao debate sobre as recentes mudanças na política de atenção primária propostas pelo MS. Abrasco. [2019]. [acesso em 2020 maio 28]. Disponível em: https://www.abrasco.org.br/site/wp-content/uploads/2019/10/Contribuicoesdospesquisaadores_REDEAPS.pdf.

7. Giovanella L, Franco CM, Almeida PF. Política Nacional de Atenção Básica: para onde vamos? Ciênc. e Saúde Colet. 2020 [acesso em 2020 abr 25]; 25(4):1475-82. Disponível em: http://orcid.org/0000-0003-1430-6951.

8. Rede de Pesquisa em Atenção Primária à Saúde da Abrasco. A APS no SUS no enfrentamento da pandemia COVID-19. 2020 mar 23. [acesso em 2020 maio 28]. Disponível em: https://redeaps.org.br/2020/03/23/a- -aps-no-sus-no-enfrentamento-da-pandemia-covid-19/.

9. Lorenzo SM. La pandemia COVID-19: lo que hemos aprendido hasta ahora desde España. APS EM Rev. 2020 [acesso em 2020 jul 3]; 2(1):28-32. Disponível em: https:// apsemrevista.org/aps/article/view/66.

10. Roberton T, Carter ED, Chou VB, et al. Early estimates of the indirect effects of the COVID-19 pandemic on maternal and child mortality in low-income and middle-income countries: a modelling study. Lancet Glob Heal. 2020 [acesso em 2020 maio 16]; (8):e901-8. Disponível em: https://doi.org/10.1016/S2214-109X(20)30229-1.

11. República Portuguesa. Serviço Nacional de Saúde. Diretoria Geral da Saúde. Áreas dedicadas Covid-19. [acesso em 2020 maio 16]. Disponível em: https://covid19.min-saude.pt/areas-dedicadas-covid-19/.

12. Li X, Krumholz HM, Yip W, et al. Quality of primary health care in China: challenges and recommendations. Lancet. 2020 [acesso em 2020 jul 9]; 395(10239):1802-12. Disponível em: https://www.thelancet.com/pdfs/journals/lancet/PIISO140-6736(20)30122-7.pdf.

13. Vega R. Lecciones de Cuba en la contención de la pandemia por COVID-19 desde el papel de la Atención Primaria en Salud. Microsoft Sway. 2020. [acesso em 2020 jul 9]. Disponível em: https://sway.office.com/ kMEWRcOaXcuhmJnn?ref=Link.

14. Vitória AM, Campos GWS. Só com APS forte o sistema pode ser capaz de achatar a curva de crescimento da pandemia e garantir suficiência de leitos UTI. [São Paulo]: Cosems SP; 2020. [acesso em 2020 abr 22]. Disponível em: http://www.cosemssp.org.br/wp-content/uploads/2020/04/So-APS-forte-para-ter-leitos-UTI-.pdf.

15. Engstrom E, Melo E, Giovanella L, et al. Nota Técnica. Recomendações para a organização da Atenção Primária à Saúde no SUS no enfrentamento da Covid-19. Série Linha de Cuidado Covid-19 na Rede de Atenção à Saúde. Rio de Janeiro: Observatório Covid-Fiocruz; 2020. [acesso em 2020 jul 2]. Disponível em: https://portal. fiocruz.br/sites/portal.fiocruz.br/files/documentos/ 
recomendacoes_aps_no_sus_para_enfrentamento_da_ covid-19_versao_leitura_uma_coluna__.pdf.

16. Medina MG, Giovanella L, Bousquat AEM, et al. Atenção Primária à Saúde em tempos de Covid-19: O que fazer? Cad. Saúde Pública. 2020 [acesso em 2020 ago 30]; 36(8):e00149720. Disponível em: http://cadernos.ensp. fiocruz.br/csp/artigo/1140/atencao-primaria-a-saude-em-tempos-de-covid-19-o-que-fazer.

17. Teixeira CF, Paim JS, Vilasbôas AL. SUS, modelos assistenciais e vigilância da saúde. Inf. Epidemiol. Sus. 1998 [acesso em 2020 jul 10]; 7(2):7-28. Disponível em: http://scielo.iec.gov.br/scielo.php?script=sci arttext\&pid=S0104-16731998000200002\&lng=pt.http:// dx.doi.org/10.5123/S0104-16731998000200002.

18. Teixeira CF, Solla JP. Modelo de atenção à saúde: vigilância e saúde da família. Salvador: Editora EDUFBA; 2006. [acesso em 2020 jul 9]. Disponível em: https://static.scielo.org/scielobooks/f7/pdf/teixeira-8523204008. pdf.

19. Pedebos LA, Rocha DK, Tomasi Y. A vigilância do território na atenção primária: contribuição do agente comunitário na continuidade do cuidado. Saúde debate. 2018 [acesso em 2020 jul 10]; 42(119)940-951. Disponível em: http://www.scielo.br/scielo.php?script=sci arttext\&pid=S0103-11042018000400940\&tlng=pt.

20. Nascimento MS, Nascimento MA. Prática da enfermeira no Programa de Saúde da Família: a interface da vigilância da saúde versus as ações programáticas em saúde. Ciênc. Saúde Colet. 2005; 10(2):333-345.

21. Santos S, Melo C, Dallaire C, et al. Contextual determinants of decentralization of epidemiological surveillance for the family health team. Interface. 2015; (54):443454 .

22. Santos S, Melo C, Costa H, et al. Avaliação da capacidade de gestão descentralizada da vigilância epidemiológica no estado da Bahia. Ciênc. Saúde Colet. 2012; 17(4):873882.

23. Guimarães FG, Carvalho TML, Bernardes RM, et al. A organização da atenção Primária à Saúde de Belo Ho- rizonte no enfrentamento da Pandemia Covid 19: relato de experiência. APS EM Rev. 2020 [acesso em 2020 jul 3]; 2(2):74-82. Disponível em: https://apsemrevista. org/aps/article/view/128.

24. Ruiz DC, Martufi V. Telemonitoramento durante a pandemia de COVID-19 na Clínica de Família Zilda Arns RJ. Rio de Janeiro: Rede de Pesquisa em Atenção Primária à Saúde da Abrasco; 2020. [acesso em 2020 jul 19]. Disponível em: https://redeaps.org.br/2020/07/18/telemonitoramento-durante-a-pandemia-de-covid-19-na-clinica-de-familia-zilda-arns-rj/.

25. Potter C. Zero Covid-19 deaths in Vietnam. Baltimore Estados Unidos: Outbreak Observatory. Johns Hopkins Bloomberg School of Public Health. Center for Health Security; 2020. [acesso em 2020 jul 14]. Disponível em: https://www.outbreakobservatory.org/outbreakthursday-1/7/9/2020/zero-covid-19-deaths-in-vietnam?utm source $=$ Nature+Briefing $\&$ utm $\_$campaign $=6 \mathrm{~d} 59 \mathrm{e} 2 \mathrm{cbe} 7-$ -briefing-dy-20200714\&utm medium=email\&utm term=0_c9dfd39373-6d59e2cbe7-45390226.

26. Nedel FB. Enfrentando a COVID-19: APS forte agora mais que nunca! APS EM Rev. 2020 [acesso em 2020 jul 3]; 2(1):11-6. Disponível em: https://apsemrevista. org/aps/article/view/68.

27. Cheng KK, Lam TH, Leung CC. Comment Wearing face masks in the community during the COVID-19 pandemic: altruism and solidarity. Lancet. 2020. [acesso em 2020 maio 28]. Disponível em: https://www.thelancet. com/journals/lancet/article/PIIS0140-6736(20)309181/fulltext.

28. Silveira JPM, Zonta R. Experiência de reorganização da APS para o enfrentamento da COVID-19 em Florianópolis. APS EM Rev. 2020 [acesso em 2020 jul 3]; 2(2):916. Disponível em: https://apsemrevista.org/aps/article/ view/122.

29. Vale EP, Rodrigues GM, Costa DP, et al. Reorganização da Rede de Atenção à Saúde para o enfrentamento da COVID-19 no município de Canaã dos Carajás, Pará. APS EM Rev. 2020 [acesso em 2020 jul 3]; 2(2):83-90. Disponível em: https://apsemrevista.org/aps/article/ view/101. 
30. Fernandez MV, Castro DM, Fernandes LDMM, et al. Reorganizar para avançar: a experiência da Atenção Primária à Saúde de Nova Lima/MG no enfrentamento da pandemia da Covid-19. APS EM Rev. 2020 [acesso em 2020 jul 3]; 2(2):114-21. Disponível em: https://apsemrevista.org/aps/article/view/84.

31. Ribeiro MA, Júnior DGA, Cavalcante ASP, et al. (RE) Organização da Atenção Primária à Saúde para o enfrentamento da COVID-19: Experiência de Sobral-CE. APS EM Rev. 2020 [acesso em 2020 jul 3]; 2(2):177-88. Disponível em: https://apsemrevista.org/aps/article/ view/125.

32. Cardona Júnior AHS, Andrade CWQ, Caldas LNM. Educação em saúde: programa e canal de comunicação via WhatsApp da unidade básica de saúde do N6 para comunidade rural do sertão pernambucano. APS EM Rev. 2020 [acesso em 2020 jul 3]; 2(2):137-41. Disponível em: https://apsemrevista.org/aps/article/view/92.

33. Fillis MMA, Dellaroza MSG, Machado RA, et al. Saúde do trabalhador em tempos de COVID-19: a experiência do município de Londrina. APS EM Rev. 2020 [acesso em 2020 jul 3]; 2(2):106-13. Disponível em: https://apsemrevista.org/aps/article/view/97.

34. Oliveira MAB, Monteiro LDS, Oliveira RDC, et al. A prática do núcleo de apoio à saúde da família do Recife no enfrentamento à pandemia COVID-19. APS EM Rev. 2020 [acesso em 2020 jul 3]; 2(2):142-50. Disponível em: https://apsemrevista.org/aps/article/view/96.

35. Daumas RP, Silva GA, Tasca R, et al. O papel da atenção primária na rede de atenção à saúde no Brasil: limites e possibilidades no enfrentamento da COVID-19. Cad. Saúde Pública. 2020; 36(6):e00104120.

36. Ruiz DC, Martufi V. Enfrentando a pandemia no Complexo do Alemão: ações conjuntas do GT Comunitário da Clínica de Família Zilda Arns e do Gabinete de Crise do Alemão, município do Rio de Janeiro. Rio de Janeiro: Rede de Pesquisa em Atenção Primária à Saúde da Abrasco; 2020. [acesso em 2020 jul 10]. Disponível em: https:// redeaps.org.br/2020/07/06/enfrentando-a-pandemia-no-complexo-do-alemao-acoes-conjuntas-do-gt-comunitario-da-clinica-de-familia-zilda-arns-e-do-gabinete-de-crise-do-alemao-municipio-do-rio-de-janeiro/.

37. Gomes Junior AS, Latge PK, Oliveira RAT, et al. A experiência de Niterói no enfrentamento da COVID 19: notas preliminares sobre a articulação de políticas sociais e de saúde. APS EM Rev. 2020 [acesso em 2020 jul 3]; 2(2):128-136. Disponível em: https://apsemrevista.org/ aps/article/view/126.

38. Santos ABS, França MVS, Santos JLF. Atendimento remoto na APS no contexto da COVID-19: a experiência do Ambulatório da Comunidade da Escola Bahiana de Medicina e Saúde Pública em Salvador, Bahia. APS EM Rev. 2020 [acesso em 2020 jul 3]; 2(2):189-96. Disponível em: https://doi.org/10.14295/aps.v2i2.120.

39. Rodrigues AP, Felipe CR, Lima DB, et al. Telemonitoramento como estratégia de cuidado longitudinal a grupos prioritários em tempos da COVID-19: uma experiência na atenção primária à saúde do município de Vitória-ES. APS EM Rev. 2020 [acesso em 2020 jul 3]; 2(2):189-96. Disponível em: https://apsemrevista.org/aps/article/ view/100.

40. Cruz NMLV, Souza EB, Sampaio CSF, et al. Apoio psicossocial em tempos de COVID-19: experiências de novas estratégias de gestão e ajuda mútua no sul da Bahia, Brasil. APS EM Rev. 2020 [acesso 2020 jul 3]; 2(2):97105. Disponível em: https://apsemrevista.org/aps/article/view/94.

41. Fundação Oswaldo Cruz; Conselho Nacional de Saúde. Atenção primária e sistemas universais de saúde: compromisso indissociável e direito humano fundamental. Saúde debate. 2018; 42(esp1):434-451.

Recebido em 24/07/2020

Aprovado em 24/09/2020

Conflito de interesses: inexistente

Suporte financeiro: não houve 